\title{
Low-energy theorems of QCD and bulk viscosity at finite temperature and baryon density in a magnetic field
}

\author{
N.O. Agasian ${ }^{1, *}$ \\ 1 Institute of Theoretical and Experimental Physics, \\ 117218, Moscow, Russia
}

\begin{abstract}
The nonperturbative QCD vacuum at finite temperature and a finite baryon density in an external magnetic field is studied. Equations relating nonperturbative condensates to the thermodynamic pressure for $T \neq 0, \mu_{q} \neq 0$ and $H \neq 0$ are obtained, and low-energy theorems are derived. A bulk viscosity $\zeta(T, \mu, H)$ is expressed in terms of basic thermodynamical quantities describing the quark-gluon matter at $T \neq 0, \mu_{q} \neq 0$, and $H \neq 0$. Various limiting cases are also considered.
\end{abstract}

PACS:11.10.Wx,11.15.Ha,12.38.Gc,12.38.Mh

\section{INTRODUCTION}

Quantum chromodynamics (QCD) as a theory that describes strong interaction physics is still vigorously developing. Over the past few decades, particular attention has been drawn to the investigation of the behavior of strongly interacting matter under the effect of various external fields. In the real world, these are primarily temperature and the baryon density. Interest in the behavior of matter under extreme conditions (high temperature is the one starting from a characteristic QCD scale, $T \sim 200 \mathrm{MeV}$, and high baryon densities starts from $n>n_{0} \simeq 0.17 \mathrm{fm}^{-3}$, where $n_{0}$ - is a normal nuclear density) is motivated primarily by the experiments studying heavy-ion collisions. Due to this, one can expect that such experiments probe densities and temperature at which a phase transition to quark-gluon plasma, which is a new state of strongly interacting matter, is possible.

In recent years, the phase structure of the vacuum in an external magnetic field $H$ has become yet another important object of investigations. It was shown recently that magnetic fields of strength in the range $e H \sim 10 \div 10^{4} \mathrm{MeV}^{2}$ can be generated in heavy-ion collisions

* Electronic address: agasian@itep.ru 
[1-3]. Such field can lead to observable phenomena (so-called "chiral magnetic effect") [1, 47] in experiments at the Relativistic Heavy Ion Collider (RHIC) and at the Large Hadron Collider (LHC). Magnetic fields of the order of $e H \sim \Lambda_{Q C D}^{2}$ or even stronger could exist in the early Universe. Such magnetic field strengths can lead to new interesting phenomena accompanying the QCD phase transition [8-32].

In [33], the magnetic-field dependence of the quark condensate was studied on the basis of the Nambu-Jona-Lasinio model. In QCD, the one-loop result for the $H$ dependence of $\langle\bar{q} q\rangle$ was obtained in [34]. In both cases, the condensate was found to grow with increasing $H$. It follows that a naive analogy with superconductivity theory, where a magnetic field breaks the condensate of Cooper pairs, is inapplicable here. Strictly speaking, the behavior of the gluon condensate $\left\langle G^{2}\right\rangle$ in an Abelian magnetic field is also nontrivial. Gluons do not carry an electric charge, but virtual quarks generated by them shift the quantity $\left\langle G^{2}\right\rangle$ owing to their interaction with the magnetic field $H$. This phenomenon was studied in [35] making use of the low-energy theorems. The phase structure of QCD vacuum in an Abelian magnetic field at finite temperature was studied in [8, 15, 17, 19, 28, 29, 36, 37].

Relations arising from the symmetry properties play an important role in quantum field theories. Searches for symmetries and constraints that these symmetries impose on the physical properties of the system become particularly important in QCD as a theory that involves confinement, where composite states (hadrons) appear to be "observables". Lowenergy theorems, or Ward identities (scale and chiral), play a fundamental role in obtaining deeper insight into the nonperturbative vacuum properties of QCD. In QCD, low-energy theorems were obtained in the early 1980s [38]. The low-energy theorems of QCD, which follow from general symmetry properties and which are independent of the details of the confinement mechanism, make it possible to obtain information that is sometimes inaccessible within any other method. Also, they can be used as "physically reasonable" constraints in constructing effective theories and various models of the QCD vacuum. In QCD, the lowenergy theorems for $T \neq 0$ and $\mu_{q} \neq 0$ were obtained in [39, 40]. An important application of low-energy theorems in a hot QCD was obtained in [41, 42]. Based on Kubo equation and low-energy theorems, the bulk viscosity of the quark-gluon matter was shown to be directly connected to a bilocal correlator of the energy-momentum tensor, and its value for a hot QCD case was evaluated [41, 42].

A method that makes it possible to generalize the low-energy theorems of QCD to the 
case of finite temperature, a finite baryon chemical potential, and a nonzero magnetic field is developed in the present study. This method is used to study the nonperturbative vacuum and to derive an expression for a bilocal correlation function for the energy-momentum tensor and bulk viscosity in QCD for $T \neq 0, \mu_{q} \neq 0$ and $H \neq 0$.

\section{LOW-ENERGY THEOREMS OF QCD AT FINITE $T, \mu$ AND $H$}

In the Euclidean formulation, the QCD partition function in presence of an external Abelian field $A_{\mu}$ can be represented in the form (here, $T=1 / \beta$ is temperature)

$$
Z=\exp \left\{-\frac{1}{4 e^{2}} \int_{0}^{\beta} d x_{4} \int_{V} d^{3} x F_{\mu \nu}^{2}\right\} \int[D B][D \bar{q}][D q] \exp \left\{-\int_{0}^{\beta} d x_{4} \int_{V} d^{3} x \mathcal{L}\right\}
$$

where the QCD Lagrangian in a background field has the form

$$
\mathcal{L}=\frac{1}{4 g_{0}^{2}}\left(G_{\mu \nu}^{a}\right)^{2}+\sum_{q=u, d, \ldots} \bar{q}\left[\gamma_{\mu}\left(\partial_{\mu}-i Q_{q} A_{\mu}-i \frac{\lambda^{a}}{2} B_{\mu}^{a}\right)+m_{0 q}+\mu_{q} \gamma_{0}\right] q
$$

Here, $Q_{q}$ is the charge matrix for quarks of flavor $q=(u, d, s, \ldots)$ and bare mass $m_{0 q}$ and $\mu_{q}$ is the quark chemical potential; the ghost and gauge-fixing terms are not written down explicitly in order to avoid encumbering the presentation. The pressure in the system (minus a thermodynamical potential) is determined by the expression $\beta V P_{0}\left(T, \mu_{q}, H, m_{0 q}\right)=\ln Z$. From the partition function in Eq. (1), one can obtain the following expression for the gluon condensate $\left\langle G^{2}\right\rangle \equiv\left\langle\left(G_{\mu \nu}^{a}\right)^{2}\right\rangle$ :

$$
\left\langle G^{2}\right\rangle\left(T, \mu_{q}, H, m_{0 q}\right)=-4 \frac{\partial P_{0}}{\partial\left(1 / g_{0}^{2}\right)} .
$$

The system described by the partition function in (1) is characterized by the set of dimensional parameters $\Lambda_{0}, \mu_{q}, T, H, m_{0 q}\left(\Lambda_{0}\right)$ and by the dimensionless charge $g_{0}^{2}\left(\Lambda_{0}\right)$, where the bare quark masses $m_{0 q}$ and the coupling constant $g_{0}^{2}$ are specified at the scale of the ultraviolet-cutoff mass $\Lambda_{0}$. On the other hand, we can go over to the renormalized (physical) pressure $P$ and, with the aid of the properties of the renormalization group invariance of $P$, recast expression (3) into a form that involves derivatives with respect to the physical parameters $T, \mu_{q}$, and $H$ and with respect to the renormalized masses $m_{q}$.

The dimensional transmutation phenomenon leads to the appearance of the nonperturbative dimensional parameter

$$
\Lambda=\Lambda_{0} \exp \left\{\int_{\alpha_{s}\left(\Lambda_{0}\right)}^{\infty} \frac{d \alpha_{s}}{\beta\left(\alpha_{s}\right)}\right\}
$$


where $\Lambda_{0}$ is the ultraviolet-cutoff mass, $\alpha_{s}=g_{0}^{2} / 4 \pi$ and $\beta\left(\alpha_{s}\right)=d \alpha_{s}\left(\Lambda_{0}\right) / d \ln \Lambda_{0}$ is theGellMann-Low function. The quark mass $m_{0 q}$ has the anomalous dimension $\gamma_{m_{q}}$ and depends on the scale $\Lambda_{0}$. The renormalization group equation for the running mass $m_{0 q}\left(\Lambda_{0}\right)$ has the form $d \ln m_{0 q} / d \ln \Lambda_{0}=-\gamma_{m_{q}}$, and we use the modified minimal-subtraction $\overline{M S}$ scheme, where $\beta$ and $\gamma_{m_{q}}$ are independent of the quark mass. The expression for the renormalization group invariant mass then has the form

$$
m_{q}=m_{o q}\left(\Lambda_{0}\right) \exp \left\{\int^{\alpha_{s}\left(\Lambda_{0}\right)} \frac{\gamma_{m_{q}}\left(\alpha_{s}\right)}{\beta\left(\alpha_{s}\right)} d \alpha_{s}\right\} .
$$

Since the physical (renormalized) pressure is a renormalization group invariant quantity, its anomalous dimension is zero. Thus, $P$ has only a normal (canonical) dimension equal to four. Employing the renormalization group invariance of the quantity $\Lambda$, we can write $P$ in the most general form as

$$
P=\Lambda^{4} f\left(\frac{T}{\Lambda}, \frac{\mu_{q}}{\Lambda}, \frac{H}{\Lambda^{2}}, \frac{m_{q}}{\Lambda}\right)
$$

where $f$ is a function of the dimensionless ratios $T / \Lambda, \ldots$ From (4),(5) and (6) we then obtain

$$
\begin{gathered}
\frac{\partial P}{\partial\left(1 / g_{0}^{2}\right)}=\frac{\partial P}{\partial \Lambda} \frac{\partial \Lambda}{\partial\left(1 / g_{0}^{2}\right)}+\sum_{q} \frac{\partial P}{\partial m_{q}} \frac{\partial m_{q}}{\partial\left(1 / g_{0}^{2}\right)}, \\
\frac{\partial m_{q}}{\partial\left(1 / g_{0}^{2}\right)}=-4 \pi \alpha_{s}^{2} m_{q} \frac{\gamma_{m_{q}}\left(\alpha_{s}\right)}{\beta\left(\alpha_{s}\right)} .
\end{gathered}
$$

Further, The anomaly in the trace of the energy-momentum tensor in QCD is related to the gluon condensate by the equation

$$
\left\langle\theta_{\mu \mu}^{g}\right\rangle=\frac{\beta\left(\alpha_{s}\right)}{16 \pi \alpha_{s}^{2}}\left\langle G^{2}\right\rangle
$$

Taking into account (3), we obtain the gluon part of the trace of the energy-momentum tensor in the form

$$
\left\langle\theta_{\mu \mu}^{g}\right\rangle=\left(T \frac{\partial}{\partial T}+\sum_{q} \mu_{q} \frac{\partial}{\partial \mu_{q}}+2 H \frac{\partial}{\partial H}+\sum_{q}\left(1+\gamma_{m_{q}}\right) m_{q} \frac{\partial}{\partial m_{q}}-4\right) P .
$$

Here and below, the energy-momentum tensor $\left\langle\theta_{\mu \mu}\right\rangle$, the condensates $\left\langle G^{2}\right\rangle$ and $\langle\bar{q} q\rangle$, and the thermodynamic pressure $P$ are functions of $T, \mu_{q}, H$ and $m_{q}$.

In the one-loop approximation, we have $\beta\left(\alpha_{s}\right) \rightarrow-b \alpha_{s}^{2} / 2 \pi$ and $1+\gamma_{m_{q}} \rightarrow 1$, where $b=\left(11 N_{c}-2 N_{f}\right) / 3$. Thus, the gluon and quark parts of the trace of the energy-momentum 
tensor in hot and dense QCD in a magnetic field can be expressed in terms of the physical pressure in the one-loop approximation as

$$
\begin{gathered}
\left\langle\theta_{\mu \mu}^{g}\right\rangle=-\frac{b}{32 \pi^{2}}\left\langle G^{2}\right\rangle=\left(T \frac{\partial}{\partial T}+\sum_{q} \mu_{q} \frac{\partial}{\partial \mu_{q}}+2 H \frac{\partial}{\partial H}+\sum_{q} m_{q} \frac{\partial}{\partial m_{q}}-4\right) P, \\
\left\langle\theta_{\mu \mu}^{q}\right\rangle=\sum_{q} m_{q}\langle\bar{q} q\rangle=-\sum_{q} m_{q} \frac{\partial P}{\partial m_{q}} .
\end{gathered}
$$

In the vacuum, that is, at $T=0, \mu_{q}=0$ and $H=0$, we arrive at the well-known expression for the nonperturbative vacuum energy density. In the one-loop approximation, this expression has the form

$$
\begin{gathered}
\varepsilon_{\mathrm{vac}}=\frac{1}{4}\left\langle\theta_{\mu \mu}^{g}+\theta_{\mu \mu}^{q}\right\rangle_{0}=-P\left(T=0, \mu_{q}=0, H=0, m_{q}\right) \\
=-\frac{b}{128 \pi^{2}}\left\langle G^{2}\right\rangle_{0}+\frac{1}{4} \sum_{q} m_{q}\langle\bar{q} q\rangle_{0} .
\end{gathered}
$$

By using the relations presented above, one can derive low-energy theorems of QCD at finite temperature and finite density in the presence of a magnetic field. Strictly speaking, the $\beta$-function depends on $H$, so that the low-energy theorems could involve electromagnetic corrections, which are proportional to $\propto e^{4}$, but, since the physical pressure is independent of the scale $\Lambda_{0}$ at which ultraviolet divergencies are regularized, one can choose an ultraviolet scale in such a way that $\Lambda_{0} \gg H, T, \mu_{q}, \Lambda$. We can then restrict ourselves to the lowest order in the expansion of the $\beta$-function, with the result that electromagnetic corrections disappear. Taking this into account, we consider the trace of the energy-momentum tensor in hot and dense QCD in the one-loop approximation,

$$
\theta_{\mu \mu}(x)=-\frac{b}{32 \pi^{2}}\left(G_{\mu \nu}^{a}(x)\right)^{2}+\sum_{q} m_{q} \bar{q} q(x) .
$$

Also, we introduce the operator $\hat{D}$, defining it as

$$
\hat{D}=T \frac{\partial}{\partial T}+\sum_{q} \mu_{q} \frac{\partial}{\partial \mu_{q}}+2 H \frac{\partial}{\partial H} .
$$

From relations (11) and (12), we find for the total vacuum expectation value of the trace of the energy-momentum tensor with allowance for massive quarks that

$$
\left\langle\theta_{\mu \mu}\right\rangle=\left\langle\theta_{\mu \mu}^{g}+\theta_{\mu \mu}^{q}\right\rangle=-\frac{b}{32 \pi^{2}}\left\langle G^{2}\right\rangle+\sum_{q} m_{q}\langle\bar{q} q\rangle=(\hat{D}-4) P .
$$


Differentiating Eq.(3) $n$ times with respect to $\left(1 / g_{0}^{2}\right)$ and taking into account relations (6), (14), (15) and (16), we obtain

$$
\begin{gathered}
(\hat{D}-4)^{n+1} P=(\hat{D}-4)^{n}\left\langle\theta_{\mu \mu}^{g}(0)\right\rangle \\
=\int d^{4} x_{n} \ldots \int d^{4} x_{1}\left\langle\theta_{\mu \mu}^{g}\left(x_{n}\right) \ldots \theta_{\mu \mu}^{g}\left(x_{1}\right) \theta_{\mu \mu}^{g}(0)\right\rangle .
\end{gathered}
$$

To the right-hand side of (17) only connected diagrams are included, as usual.

Similar arguments apply to an arbitrary operator $\hat{O}(x)$ constructed from quark or gluon fields; that is,

$$
\begin{aligned}
& \left(T \frac{\partial}{\partial T}+\sum_{q} \mu_{q} \frac{\partial}{\partial \mu_{q}}+2 H \frac{\partial}{\partial H}-d\right)^{n}\langle\hat{O}\rangle \\
= & \int d^{4} x_{n} \ldots \int d^{4} x_{1}\left\langle\theta_{\mu \mu}^{g}\left(x_{n}\right) \ldots \theta_{\mu \mu}^{g}\left(x_{1}\right) \hat{O}(0)\right\rangle,
\end{aligned}
$$

where $d$ is the canonical dimension of the operator $\hat{O}$. If the operator $\hat{O}$ has an anomalous dimension as well, it is necessary to take into account the corresponding $\gamma$-function.

Let us now consider the case of $n=1$, which is of importance for physical applications. In other words, we will examine a bilocal correlation function for the tensors of the energymomentum density in hot and dense QCD in a magnetic field. In terms of this correlation function, one can express the bulk viscosity of quark-gluon plasma in a magnetic field. Then, for gluon and quark contributions to the bilocal correlator of the trace of the energymomentum tensor, we have the following relations:

$$
\begin{aligned}
\int d^{4} x\left\langle\theta_{\mu \mu}^{g}(x) \theta_{\mu \mu}^{g}(0)\right\rangle & =(\hat{D}-4)\left\langle\theta_{\mu \mu}^{g}\right\rangle, \\
\int d^{4} x\left\langle\theta_{\mu \mu}^{g}(x) \theta_{\mu \mu}^{q}(0)\right\rangle & =(\hat{D}-3)\left\langle\theta_{\mu \mu}^{q}\right\rangle .
\end{aligned}
$$

Hence, for the the bilocal correlator of the trace of the energy-momentum tensor

$$
\begin{gathered}
\Pi=\int d^{4} x\left\langle\theta_{\mu \mu}(x) \theta_{\mu \mu}(0)\right\rangle \\
=\int d^{4} x\left\langle\theta_{\mu \mu}^{g} \theta_{\mu \mu}^{g}\right\rangle+2 \int d^{4} x\left\langle\theta_{\mu \mu}^{g} \theta_{\mu \mu}^{q}\right\rangle+O\left(m_{q}^{2}\right),
\end{gathered}
$$

where we included a correlator of quark summands to $O\left(m_{q}^{2}\right)$, being proportional to a quark mass squared; in what follows we will not take it into account. Based on relations (20), we find

$$
\Pi=(\hat{D}-4)\left\langle\theta_{\mu \mu}^{g}\right\rangle+2(\hat{D}-3)\left\langle\theta_{\mu \mu}^{q}\right\rangle=(\hat{D}-4)\left\langle\theta_{\mu \mu}\right\rangle+(\hat{D}-2)\left\langle\theta_{\mu \mu}^{q}\right\rangle .
$$




\section{BULK VISCOSITY $\zeta(T, \mu, H)$}

As it was shown in [42], according to a general Kubo formula, a bulk viscosity can be evaluated as a static limit of a bilocal correlator of the trace of the energy-momentum tensor.

$$
\zeta=\frac{1}{9} \lim _{\omega \rightarrow 0} \frac{1}{\omega} \int_{0}^{\infty} d t \int d^{3} r e^{i \omega t}\left\langle\left[\theta_{\mu \mu}(x), \theta_{\mu \mu}(0)\right]\right\rangle
$$

One can introduce a spectral density expressed in terms of a retarded Green function for the trace of the energy-momentum tensor

$$
\rho(\omega, \mathbf{p})=-\frac{1}{\pi} \operatorname{Im} G^{R}(\omega, \mathbf{p})
$$

Then, as was suggested in [42], for small frequencies the spectral density has the following form:

$$
\frac{\rho(\omega, \mathbf{0})}{\omega}=\frac{9 \zeta}{\pi} \frac{\omega_{0}^{2}}{\omega_{0}^{2}+\omega^{2}},
$$

where the $\omega_{0}$ parameter determines a scale at which a perturbation theory becomes applicable. Using this ansatz, the bulk viscosity can be written as

$$
9 \zeta \omega_{0}=2 \int_{0}^{\infty} \frac{\rho(u, \mathbf{0})}{u} d u=\int d^{4} x\left\langle\theta_{\mu \mu}(x) \theta_{\mu \mu}(0)\right\rangle=\Pi .
$$

Thus, a problem to find the bulk viscosity $\zeta$ reduces to a problem to evaluate the bilocal correlator $\Pi$.

We extract from the correlator $\Pi$ a vacuum term. For this purpose, we write the following expression for the total pressure

$$
P=-\varepsilon_{\mathrm{vac}}+P_{*},
$$

where $P_{*}$ is the pressure pure thermodynamical part. Quark and gluon contributions to the trace of the energy-momentum tensor can be written as

$$
\begin{gathered}
\left\langle\theta_{\mu \mu}^{q}\right\rangle=\left\langle\theta_{\mu \mu}^{q}\right\rangle_{0}+\left\langle\theta_{\mu \mu}^{q}\right\rangle_{*}=\sum_{q} m_{q}\langle\bar{q} q\rangle_{0}+\sum_{q} m_{q}\langle\bar{q} q\rangle_{*} \\
\left\langle\theta_{\mu \mu}^{g}\right\rangle=\left\langle\theta_{\mu \mu}^{g}\right\rangle_{0}+\left\langle\theta_{\mu \mu}^{g}\right\rangle_{*}
\end{gathered}
$$

and, using equation (16)

$$
\left\langle\theta_{\mu \mu}\right\rangle=\left\langle\theta_{\mu \mu}^{q}+\theta_{\mu \mu}^{g}\right\rangle=4 \varepsilon_{\mathrm{vac}}+(\hat{D}-4) P_{*}
$$


Allowing for the thermodynamic relation

$$
\left(T \frac{\partial}{\partial T}+\sum_{q} \mu_{q} \frac{\partial}{\partial \mu_{q}}-4\right) P=\varepsilon-3 P
$$

and taking into consideration the magnetic moment $M=\partial P / \partial H$ we have

$$
\left\langle\theta_{\mu \mu}\right\rangle=4 \varepsilon_{\mathrm{vac}}+(\varepsilon-3 P)_{*}+2 M H .
$$

Then the correlator $(21,22)$ can be written as

$$
\Pi=\Pi_{0}+\Pi_{*}^{q}+\Pi_{*}^{g},
$$

where the vacuum contribution

$$
\Pi_{0}=-4\left\langle\theta_{\mu \mu}\right\rangle_{0}-2\left\langle\theta_{\mu \mu}^{q}\right\rangle_{0}=-16 \varepsilon_{\mathrm{vac}}-2 \sum_{q} m_{q}\langle\bar{q} q\rangle_{0}
$$

For the quark contribution $\Pi_{*}^{q}$ we get the following expression

$$
\Pi_{*}^{q}=\left(T \frac{\partial}{\partial T}+\sum_{q} \mu_{q} \frac{\partial}{\partial \mu_{q}}+2 H \frac{\partial}{\partial H}-2\right) \sum_{q} m_{q}\langle\bar{q} q\rangle_{*}
$$

The gluonic part $\Pi_{*}^{g}$ of the correlator can be written as

$$
\begin{gathered}
\Pi_{*}^{g}=\left(T \frac{\partial}{\partial T}-4\right)(\varepsilon-3 P)_{*}+\left(\sum_{q} \mu_{q} \frac{\partial}{\partial \mu_{q}}+2 H \frac{\partial}{\partial H}\right)(\varepsilon-3 P)_{*} \\
+\left(T \frac{\partial}{\partial T}+\sum_{q} \mu_{q} \frac{\partial}{\partial \mu_{q}}+2 H \frac{\partial}{\partial H}-4\right) 2 M H .
\end{gathered}
$$

We use the following definitions of thermodynamic quantities, in terms of pressure and energy density: entropy density $s=\partial P / \partial T$, specific heat $c_{v}=\partial \varepsilon / \partial T$, velocity of sound $c_{s}^{2}=\partial P / \partial \varepsilon=s / c_{v}^{2}$, and medium magnetic susceptibility $\chi=\partial M / \partial H=\partial^{2} P / \partial H^{2}$. Then we find

$$
\begin{aligned}
& \Pi_{*}^{g}=T s\left(\frac{1}{c_{s}^{2}}-3\right)+\left(\sum_{q} \mu_{q} \frac{\partial}{\partial \mu_{q}}-4\right)(\varepsilon-3 P)_{*} \\
& +4 \chi H^{2}-12 M H+4 H\left(T \frac{\partial}{\partial T}+\sum_{q} \mu_{q} \frac{\partial}{\partial \mu_{q}}\right) M .
\end{aligned}
$$

Thus, equations (33), (34), and (36) express the bilocal correlator of the trace of the energymomentum tensor $\Pi$, and, correspondingly, in accordance with (26), of the bulk viscosity $\zeta$ via thermodynamic parameters: $T, \mu_{q}, H,\langle\bar{q} q\rangle_{*},(\varepsilon-3 P), s, c_{s}^{2}, M, \chi$ 


$$
\begin{gathered}
9 \zeta(T, \mu, H) \omega_{0}=-16 \varepsilon_{\mathrm{vac}}-2 \sum_{q} m_{q}\langle\bar{q} q\rangle_{0} \\
+\left(T \frac{\partial}{\partial T}+\sum_{q} \mu_{q} \frac{\partial}{\partial \mu_{q}}+2 H \frac{\partial}{\partial H}-2\right) \sum_{q} m_{q}\langle\bar{q} q\rangle_{*} \\
+T s\left(\frac{1}{c_{s}^{2}}-3\right)+\left(\sum_{q} \mu_{q} \frac{\partial}{\partial \mu_{q}}-4\right)(\varepsilon-3 P)_{*} \\
+4 \chi H^{2}-12 M H+4 H\left(T \frac{\partial}{\partial T}+\sum_{q} \mu_{q} \frac{\partial}{\partial \mu_{q}}\right) M .
\end{gathered}
$$

We explore various limiting cases. Let us consider $\mu_{q}=H=0$ and $T \neq 0$, which corresponds to hot QCD studied in [42]. Taking into account (13) and using PCAC relation

$$
\sum_{q} m_{q}\langle\bar{q} q\rangle_{0}=-F_{\pi}^{2} M_{\pi}^{2}-F_{K}^{2} M_{K}^{2}
$$

we write the vacuum contribution $\Pi_{0}$ as

$$
\Pi_{0}=-4\left\langle\theta_{\mu \mu}\right\rangle_{0}-2\left\langle\theta_{\mu \mu}^{q}\right\rangle_{0}=-4\left\langle\theta_{\mu \mu}^{g}\right\rangle_{0}-6\left\langle\theta_{\mu \mu}^{q}\right\rangle_{0}=16\left|\varepsilon_{\mathrm{vac}}^{g}\right|+6\left(F_{\pi}^{2} M_{\pi}^{2}+F_{K}^{2} M_{K}^{2}\right)
$$

where $\varepsilon_{\text {vac }}^{g}$ is the gluonic contribution to the vacuum energy density (13). Assigning $\mu_{q}=$ $H=0$ in (37) and allowing for (38), (39), we find for the correlator $\Pi$ and , correspondingly, for the bulk viscosity

$$
\begin{gathered}
9 \zeta(T) \omega_{0}=T s\left(\frac{1}{c_{s}^{2}}-3\right)-4(\varepsilon-3 P)_{*}+\left(T \frac{\partial}{\partial T}-2\right) \sum_{q} m_{q}\langle\bar{q} q\rangle_{*} \\
+16\left|\varepsilon_{\mathrm{vac}}^{g}\right|+6\left(F_{\pi}^{2} M_{\pi}^{2}+F_{K}^{2} M_{K}^{2}\right),
\end{gathered}
$$

which exactly conforms to the main result in [42].

Now consider the utmost case of the cold quark matter $T=H=0$ and $\mu_{q} \neq 0$. And take into account that for such a variant

$$
\varepsilon-3 P=\left(\sum_{q} \mu_{q} \frac{\partial}{\partial \mu_{q}}-4\right) P=\sum_{q} \mu_{q} n_{q}-4 P,
$$

where $n_{q}=\partial P / \partial \mu_{q}$ is the quark density. We use relation (28) and definition $P(\mu)=$ $-\varepsilon_{\mathrm{vac}}+P_{*}$, then we suppose $T=H=0$ in (37), and, after simple transformations, we find ${ }^{1}$

\footnotetext{
${ }^{1}$ The expression obtained for the bulk viscosity of the cold quark matter (42) does not coincide with a corresponding result in [43].
} 


$$
9 \zeta(\mu) \omega_{0}=16 P(\mu)-2 \sum_{q} m_{q}\langle\bar{q} q\rangle+\sum_{q} m_{q} \mu_{q} \frac{\partial\langle\bar{q} q\rangle}{\partial \mu_{q}}-7 \sum_{q} \mu_{q} n_{q}+\sum_{q, q^{\prime}} \mu_{q} \mu_{q^{\prime}} \frac{\partial^{2} P(\mu)}{\partial \mu_{q} \partial \mu_{q^{\prime}}}
$$

We note that in (42) an expression for the all quark condensate $\langle\bar{q} q\rangle$ including vacuum term is entering.

In the limiting case of hot and dense quark-gluon matter, an expression for $\zeta(T, \mu, H=0)$ is obtained immediately from equation (37) for $\zeta(T, \mu, H)$ by substituting $H=0$.

\section{CONCLUSIONS}

According to Eq. (37), the bulk viscosity $\zeta$ in a magnetic field is proportional to the magnetic susceptibility $\chi$. In two different phases of strongly interacting matter (hadron and quark-gluon), the magnetic susceptibility has different signs [8]. This is because hadron matter at temperatures below the quark-hadron phase transition primarily consists of a gas of hot $\pi$-mesons, which in the magnetic field behaves as a diamagnetic medium of spinless charged particles. The system above the phase transition point is in the paramagnetic phase of hot quarks and gluons. This significantly affects the bulk viscosity of strongly interacting matter in the magnetic field, which should have a jump at the transition point, leading to interesting observable phenomena.

In this paper a nonperturbative QCD vacuum at a finite temperature and a finite baryon chemical potential in an external magnetic field are considered. Relations between nonperturbative condensates and thermodynamical pressure at $T \neq 0, \mu_{q} \neq 0$, and $H \neq 0$ are obtained, and low-energy theorem are derived. A common formula for the bulk viscosity of the quark-gluon medium at $T \neq 0, \mu_{q} \neq 0$, and $H \neq 0$, is derived, which links $\zeta(T, \mu, H)$ to thermodynamic system parameters: entropy density $s=\partial P / \partial T$, velocity of sound $c_{s}^{2}=\partial P / \partial \varepsilon=s / c_{v}^{2}$, non-ideality $(\varepsilon-3 P)$, quark condensate $\langle\bar{q} q\rangle_{*}$, and medium magnetic susceptibility $\chi=\partial M / \partial H=\partial^{2} P / \partial H^{2}$. Some physically interesting limiting cases for the bulk viscosity are considered. These phenomena and exact relations obtained here may prove to be of importance in examining the quark-hadron phase transition in heavy ion collisions and in the early Universe, where strong magnetic fields are generated.

I am grateful to A.B. Kaidalov, V.A. Novikov, and Yu.A. Simonov for useful discussions about the problems considered in the present study. 


\section{REFERENCES}

1. D. E. Kharzeev, L. D. McLerran and H. J. Warringa, Nucl. Phys. A 803, 227 (2008).

2. V. Skokov, A. Y. Illarionov and V. Toneev, Int. J. Mod. Phys. A 24, 5925 (2009)

3. V. Voronyuk, V. D. Toneev, W. Cassing, E. L. Bratkovskaya, V. P. Konchakovski and S. A. Voloshin, Phys. Rev. C 83, 054911 (2011)

4. D. Kharzeev, R. D. Pisarski and M. H. G. Tytgat, Phys. Rev. Lett. 81, 512 (1998).

5. D. Kharzeev, Phys. Lett. B 633, 260 (2006).

6. K. Fukushima, D. E. Kharzeev and H. J. Warringa, Phys. Rev. D 78, 074033 (2008).

7. K. Fukushima, M. Ruggieri and R. Gatto, Phys. Rev. D 81, 114031 (2010).

8. N. O. Agasian and S. M. Fedorov, Phys. Lett. B 663, 445 (2008).

9. E. S. Fraga and A. J. Mizher, Phys. Rev. D 78, 025016 (2008).

10. E. S. Fraga and A. J. Mizher, Nucl. Phys. A 820, 103C (2009).

11. A. J. Mizher and E. S. Fraga, Nucl. Phys. A 831, 91 (2009).

12. P. V. Buividovich, M. N. Chernodub, E. V. Luschevskaya and M. I. Polikarpov, Phys. Lett. B 682, $484(2010)$.

13. A. Ayala, A. Bashir, A. Raya and A. Sanchez, Phys. Rev. D 80, 036005 (2009).

14. P. V. Buividovich, M. N. Chernodub, E. V. Luschevskaya and M. I. Polikarpov, Phys. Rev. D 80, 054503 (2009).

15. A. J. Mizher, M. N. Chernodub and E. S. Fraga, Phys. Rev. D 82, 105016 (2010).

16. M. D'Elia, S. Mukherjee and F. Sanfilippo, Phys. Rev. D 82, 051501 (2010).

17. R. Gatto and M. Ruggieri, Phys. Rev. D 82, 054027 (2010).

18. M. N. Chernodub, Phys. Rev. D 82, 085011 (2010).

19. M. Ruggieri, AIP Conf. Proc. 1317, 360 (2011), [arXiv:1009.2342 [hep-ph]].

20. S. Fayazbakhsh and N. Sadooghi, Phys. Rev. D 83, 025026 (2011).

21. R. Gatto and M. Ruggieri, Phys. Rev. D 83, 034016 (2011).

22. M. Ruggieri, PoS FACESQCD, 019 (2010), [arXiv:1102.1832 [hep-ph]].

23. M. Frasca and M. Ruggieri, Phys. Rev. D 83, 094024 (2011)

24. M. D'Elia and F. Negro, Phys. Rev. D 83, 114028 (2011). 
25. J. O. Andersen and R. Khan, Phys. Rev. D 85, 065026 (2012).

26. J. O. Andersen, arXiv:1202.2051 [hep-ph].

27. J. O. Andersen and A. Tranberg, arXiv:1204.3360 [hep-ph].

28. G. S. Bali et al., arXiv:1111.4956 [hep-lat]; arXiv:1111.5155 [hep-lat].

29. V. V. Braguta, P. V. Buividovich, T. Kalaydzhyan, S. V. Kuznetsov and M. I. Polikarpov, PoS LATTICE2010, 190 (2010), [arXiv:1011.3795 [hep-lat]].

30. P. V. Buividovich, M. N. Chernodub, D. E. Kharzeev, T. Kalaydzhyan, E. V. Luschevskaya and M. I. Polikarpov, Phys. Rev. Lett. 105, 132001 (2010).

31. A. Gorsky, P. N. Kopnin and A. V. Zayakin, Phys. Rev. D 83, 014023 (2011).

32. B. Kerbikov, arXiv:1102.1326 [hep-ph]; B. Kerbikov and M. Andreichikov, arXiv:1107.1990 [hep-ph].

33. S. P. Klevansky and R. H. Lemmer, Phys. Rev. D 39 (1989) 3478.

34. I. A. Shushpanov and A. V. Smilga, Phys. Lett. B 402, 351 (1997).

35. N. O. Agasian and I. A. Shushpanov, JETP Lett. 70, 717 (1999); Phys. Lett. B 472, 143 (2000); JHEP 0110, 006 (2001).

36. N. O. Agasian, Phys. Lett. B 488, 39 (2000); Phys. Atom. Nucl. 64, 554 (2001); Phys. Atom. Nucl. 71, 1967 (2008).

37. A. V. Zayakin, JHEP 0807, 116 (2008).

38. V. A. Novikov, M. A. Shifman, A. I. Vainshtein, and V. I. Zakharov, Nucl. Phys. B 191, 301 (1981); Sov. J. Part. Nucl. 13, 224 (1982); A. A. Migdal and M. A. Shifman, Phys. Lett. B 114, 445 (1982).

39. P. J. Ellis, J. I. Kapusta, and H. B. Tang, Phys. Lett. B 443, 63 (1998).

40. I. A. Shushpanov, J. I. Kapusta and P. J. Ellis, Phys. Rev. C 59, 2931 (1999).

41. D. Kharzeev and K. Tuchin, JHEP 0809, 093 (2008).

42. F. Karsch, D. Kharzeev and K. Tuchin, Phys. Lett. B 663, 217 (2008).

43. M. M. Wang, Y. Jiang, B. Wang, W. M. Sun and H. S. Zong, Mod. Phys. Lett. A 26, 1797 (2011). 